\title{
Computational scrutiny of image denoising method found on DBAMF under SPN surrounding
}

\author{
Vorapoj Patanavijit \\ Department of Electrical and Electronic Engineering, Faculty of Engineering, \\ Assumption University of Thailand, Thailand
}

\begin{tabular}{l} 
Article Info \\
\hline Article history: \\
Received Sep 3, 2019 \\
Revised Feb 27, 2020 \\
Accepted Mar 8, 2020 \\
\hline Keywords: \\
AMF (adaptive median filter) \\
Digital image denoising \\
ROAD (rank-ordered absolute \\
differences) \\
SMF (standard median filter)
\end{tabular}

\begin{abstract}
Traditionally, rank order absolute difference (ROAD) has a great similarity capacity for identifying whether the pixel is SPN or noiseless because statistical characteristic of ROAD is desired for a noise identifying objective. As a result, the decision based adaptive median filter (DBAMF) that is found on ROAD technique has been initially proposed for eliminating an impulsive noise since 2010. Consequently, this analyzed report focuses to examine the similarity capacity of denoising method found on DBAMF for diverse SPN Surrounding. In order to examine the denoising capacity and its obstruction of the denoising method found on DBAMF, the four original digital images, comprised of Airplane, Pepper, Girl and Lena, are examined in these computational simulations for SPN surrounding by initially contaminating the SPN with diverse intensity. Later, all contaminated digital images are denoised by the denoising method found on DBAMF. In addition, the proposed denoised image, which is computed by this DBAMF denoising method, is confronted with the other denoised images, which is computed by standard median filter (SMF), gaussian filter and adaptive median filter (AMF) for demonstrating the DBAMF capacity under subjective measurement aspect.
\end{abstract}

Copyright $\odot 2020$ Institute of Advanced Engineering and Science. All rights reserved.

\section{Corresponding Author:}

Vorapoj Patanavijit,

Department of Electrical and Electronic Engineering,

Faculty of Engineering, Assumption University,

PKE Bldg., 2nd Flr., 88 Moo 8 Bang Na-Trad Km. 26, Bangsaothong, Samuthprakarn 10540, Thailand.

Email: patanavijit@yahoo.com

\section{RELEVANT RESEARCHED OF DENOISING METHODS FOF SPN}

Because of diverse reasons such as fault in synchronization of analog to digital process, malfunction of CCD chip, fault in addressing of storage process, and fault in transmission, etc., the impulsive noise [1-5] can be separated into two main categories: Salt and pepper noise (SPN) and random magnitude impulsive noise (RMIN) from algebraic formulation aspect. Hence, may SPN denoising methods [1-9] have been examined for more than two decades due to demanding of modern digital image applications [10-23]: retina classification, super resolution, emotion classification, etc. At first, the SMF (Standard Median Filter) [8, 24] is discovered in 1975 for excluding SPN (Salt and Pepper Noise) and, later, is become one of the most capable and practical denoising methods from the fact that this method is low computation and high capability. The denoised method based on Gaussain filter [24] is desired for well applying on Gaussain noise but this method has poor performance for SPN. Later, adaptive median filter (AMF) [7, 25], which is improved from the SMF denoising method by varing its window size, is proposed and its performance is better than the SMF denoising method. After two decades, the modern decision based adaptive median filter (DBAMF) [3, 25], which is improved from the SMF-denoising method, is discovered for denoising RMIN in 2010.

The DBAMF method is formulated from two main schemes: noise classification schemes (that is found on ROAD (Rank Order Absolute Difference)) and noise exclusion schemes (that is found on SMF [5, 25]). Unfortunately, there are no securitized researches of DBAMF capability and its constraint when this denoising 
method is implemented on SPN at diverse intensity. Consequently, this analyzed report focuses to examine the similarity capacity of denoising method found on DBAMF [3] for diverse SPN surrounding in order to analytically understand its upper bound of its performance and its limitation for future implementations.

\section{THE PRIMARY CONCEPT OF DBAMF}

The distorted portrait is mathematically explained as $\mathbf{Y}$ and the portrait intensity is mathematically explained as $y(i, j)$. The DBAMF scheme [3,25] can be separated into two primary schemes: noise recognizing scheme and noise repairing scheme, which can be comprehensively reviewed as upcoming.

\subsection{The primary concept of noise recognizing scheme}

The performing arithmetic concept of the noise recognizing scheme can be reviewed as.

- Determine the calculated square region $\underline{\mathbf{W}}_{3 \times 3}$ at $3 \times 3 \quad(w=3)$ of the processed portrait pixels at $(i, j)$ coordination.

- Determine the absolute difference $\left(D_{s, t}\left(y_{i, j}\right)\right.$ ) with normalization, so called NAD, of the processed portrait pixel with middlemost coordination $(i, j)$, which can be comprehensively clarified as upcoming.

$$
D_{s, t}\left(y_{i, j}\right)=\left|y_{i, j}-y_{s, \mathrm{t}}\right| / 255
$$

- Determine the vector of absolute difference $\left(D_{s, t}\left(y_{i, j}\right)\right.$ ) with normalization, so called NROAD (the processed portrait pixel with middlemost coordination $(i, j)$ ), which are aligned for storing only five undermost values from eigh values in the calculated square region. Later, the statistical mean of NROAD can be comprehensively clarified as upcoming.

$$
\operatorname{ROAD}_{m=5}=\frac{1}{5} \sum_{m=1}^{5} D_{s, t}\left(y_{i, j}\right)
$$

- From NROAD, if the statistical mean of NROAD, which fluctuates between 0.00 to 1.00 for all pixels in the processed portrait, is greater than a stable constant $T_{0}[3,25]$ then the processed portrait pixel is recognized as the distorted pixel, otherwise then the processed portrait pixel is recognized as the noise-free pixel. Therefore, the the Noise Detected Matrix can be comprehensively clarified as upcoming.

$$
\mathrm{NDM}=1, \text { if } \operatorname{ROAD}_{m=5} \geq T_{0} \text { otherwise } 0, \text { if } \operatorname{ROAD}_{m=5}<T
$$

From the above noise recognizing scheme of the DBAMF, we can comprehensively display this processing scheme in the upcoming flowchart as Figure 1.

\subsection{The primary concept of noise repairing scheme}

The performing arithmetic concept of the noise repairing scheme can be reviewed as.

- Determine the calculated square region $\underline{\mathbf{W}}_{3 \times 3}$ at $3 \times 3 \quad(w=3)$ of NDM (noise detected matrix) at $(i, j)$ coordination.

- From the calculated square region of NDM, if the total noise-free pixels is fewer than three pixels then the dimension of the calculated square region $\underline{\mathbf{W}}_{3 \times 3}$ is expanded by one and the Step 2 is reexecuted.

- From the calculated square region of NDM, if the total distorted pixels is more than two pixels then the repaired pixel is executed by as upcoming.

$$
\hat{Y}_{i, j}=\operatorname{median}\left\{Y_{i-s, j-t} \mid(s, t) \in W_{F}\right\}
$$

- The duplicated sheme is re executed for every pixels in the processed portrait pixels.

From the above noise repairing scheme of the DBAMF, we can comprehensively display this processing scheme in the upcoming flowchart as Figure 2. 


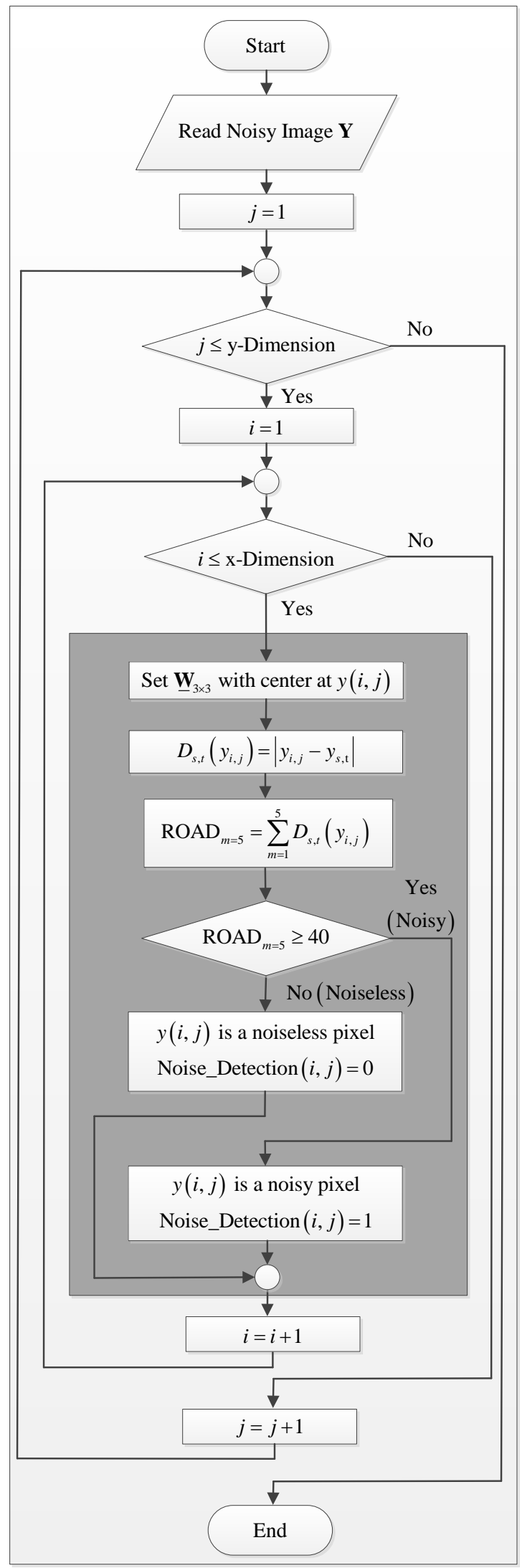

Figure 1. The arithmetic concept of the noise recognizing scheme

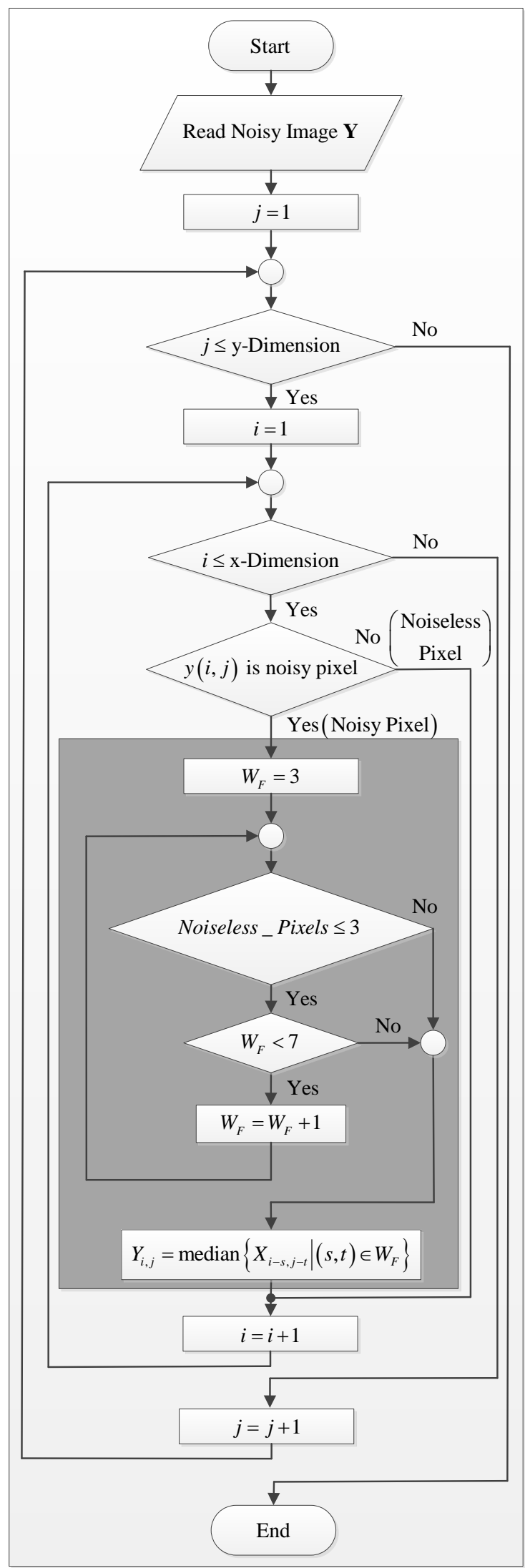

Figure 2. The arithmetic concept of the noise repairing scheme 


\section{THE COMPUTATIONAL EXAPLE OF DBAMF CONCEPT}

In first cases, this part comprehensively reviewes the calculation of the example of DBAMF noise recognizing scheme as in Figure 3(a) for obviously reviewing the processed calculation where $y_{i, j}$ is a distored pixel, which is distorted by for salt and pepper noise $\left(y_{i, j}=255\right.$ ). Later, the distored pixel is repaired as in Figure 3(b) where the distored pixel $\left(y_{i, j}=255\right.$ ) is repaired to be the repaired pixel ( $y_{i, j}=118$ ).

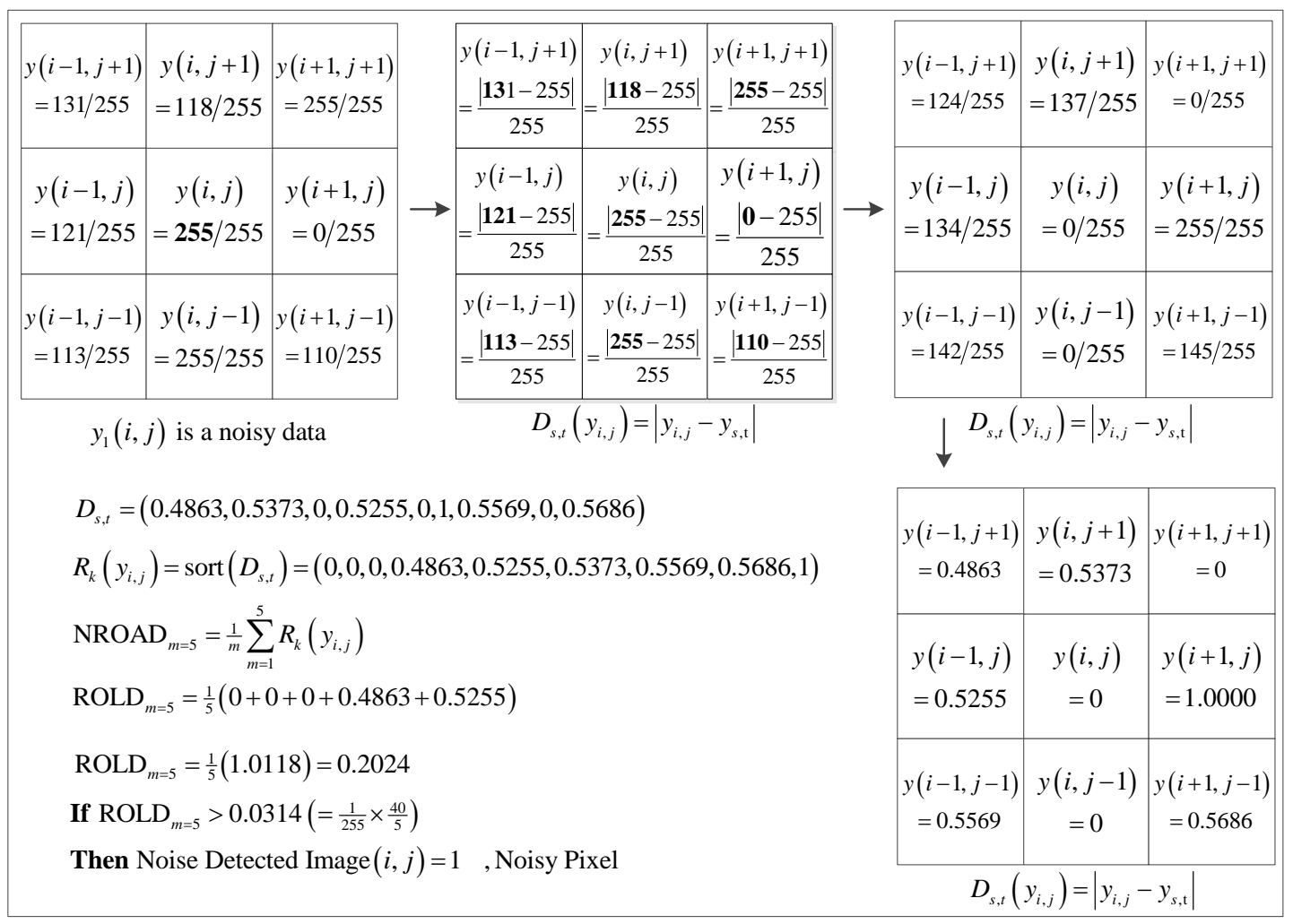

(a)

\begin{tabular}{|c|c|c|}
\hline $\begin{array}{c}y(i-1, j+1) \\
=131 / 255\end{array}$ & $\begin{array}{c}y(i, j+1) \\
=118 / 255\end{array}$ & $\begin{array}{c}y(i+1, j+1) \\
=255 / 255\end{array}$ \\
\hline $\begin{array}{c}y(i-1, j) \\
=121 / 255\end{array}$ & $\begin{array}{c}y(i, j) \\
=\mathbf{2 5 5} / 255\end{array}$ & $\begin{array}{c}y(i+1, j) \\
=0 / 255\end{array}$ \\
\hline $\begin{array}{c}y(i-1, j-1) \\
=113 / 255\end{array}$ & $\begin{array}{c}y(i, j-1) \\
=255 / 255\end{array}$ & $\begin{array}{c}y(i+1, j-1) \\
=110 / 255\end{array}$ \\
\hline
\end{tabular}

$y_{1}(i, j)$ is a noisy data

\begin{tabular}{|c|c|c|c|c|c|c|}
\hline$y(i-1, j+1)$ & $y(i, j+1)$ & $y(i+1, j+1)$ & \multirow{2}{*}{$\hat{y}(i, j)=\operatorname{modian}_{\text {noiseless pixels }}$} & {$[133$} & 118 & - \\
\hline$=0$ & $=0$ & $=1$ & & 121 & - & \\
\hline$y(i-1, j)$ & $y(i, j)$ & $y(i+1, j)$ & \multirow{4}{*}{\multicolumn{4}{|c|}{$\begin{array}{l}\hat{y}(i, j)=\operatorname{moiseless~pixels~}_{(131,118,121,113,110)} \\
\hat{y}(i, j)=\underset{\text { noiseless pixels }}{\operatorname{median}}(110,113,118,121,131) \\
\hat{y}(i, j)=118\end{array}$}} \\
\hline$=0$ & $=1$ & $=1$ & & & & \\
\hline$y(i-1, j-1)$ & $y(i, j-1)$ & $y(i+1, j-1)$ & & & & \\
\hline$=0$ & $=1$ & $=0$ & & & & \\
\hline
\end{tabular}

Figure 3 (a). The computer example of the noise recognizing scheme, (b) The computer example of the noise repairing scheme

\section{RESULTS AND DISCUSSION}

In this examining of the DBAMF denoising capacity, the calculation software in this analyzed report is MATLAB program that is run on workstation computers with the hardware detail: the CPU is Intel i7-6700HQ and the internal memory is $16 \mathrm{~GB}$ and all workstation computers simulate on diverse portraits, 
which are contained of Airplane, Pepper, Girl and Lena, at numerous SPN densities where all diverse portraits that are distorted by adding synthesized SP noise. All distorted portraits are repaired for obtaining the finest quality and best PSNR by executing the image denoising method found on DBAMF for first noise recognizing scheme (in order to recognize whether the pixel is noise-free or noisy) and, later, noise repair scheme (in order to repair only the noisy pixels).

\subsection{The experimental investigation of noise recognizing scheme}

This simulated experiment section investigates the optimized stable constant $T_{0}$ for providing the finest quality and best PSNR as shown in Table 1-4. The stable constant $T_{0}$, which fluctuates between 0.00 to 1.00 for all pixels in the processed portrait, ultimately impacts to the denoising capacity of DBAMF method. Consequently, this computer examining comprehensively determines the stable constant $T_{0}$, which make the finest quality and best PSNR when each distorted portrait is executed by denoising capacity of DBAMF method. The numerous digital portraits (which are contained of Airplane, Pepper, Girl and Lena) are used to analyze the stable constant $T_{0}$ by varying from 0.00 to 0.50 at 0.025 incremented steps as displayed in Table 1 to Table 4, respectively.

- From these computer examining of Girl in Table 1, the optimized pre-specified constant $T_{0}$ is about $0.1153 \pm 0.0005$ or be fluctuated from 0.075 to 0.150 for making the finest DBAMF denoising capacity

- From these computer examining of Pepper in Table 2, the optimized pre-specified constant $T_{0}$ is about $0.1069 \pm 0.0010$ or be fluctuated from 0.025 to 0.150 for making the finest DBAMF denoising capacity.

Table 1. The denoising capacity interconnection of PSNR and the stable constant $T_{0}$ for airplane image

\begin{tabular}{|c|c|c|c|c|c|c|c|c|c|c|c|c|c|c|c|c|c|c|}
\hline \multirow{2}{*}{$\begin{array}{l}\text { Fix } \\
\text { Con }\end{array}$} & \multicolumn{18}{|c|}{ PSNR (dB) } \\
\hline & 5 & 10 & 15 & 20 & 25 & 30 & 35 & 40 & 45 & 50 & 55 & 60 & 65 & 70 & J & 80 & 85 & \\
\hline 0.025 & 5.66 & 5.46 & 4.34 & 23.4 & .36 & .13 & .17 & & 2 & & & & & 74 & 88 & 06 & & \\
\hline & & & & & & & & & 4 & & & & & & & & & \\
\hline 5 & & & & & 1 & & & & & & & & & & & & & \\
\hline & & & & & & & & & & & & & & & & & & \\
\hline & & & & & & & & & & & & & & & & 85 & & \\
\hline & & & & & & & & & & & & & & & & 99 & & \\
\hline & & & & & & & & & & & & & & & & & & \\
\hline & & & & & & & & & & & & & & & & & & \\
\hline & & & & & & & & & & & & & & & & & & \\
\hline & & & & & & & & & & & & & & & & 2 & & \\
\hline & & & & & & & & & & & & & & & & 90 & & \\
\hline 00 & & & & & & & & & 7 & & & & & & & 6.87 & & 5 \\
\hline & & & & & & & & & 8 & & & & & & 20 & 6.72 & & 5.62 \\
\hline & & & & & & & & & 7 & & & & & & 23 & .77 & & \\
\hline & & & & & & & & & & & & & & & 7.20 & 71 & & \\
\hline & & & & & & & & & & & & & & & 97 & 54 & & 56 \\
\hline & & & & & & & & & & & & & & & & .29 & & 56 \\
\hline & & & & & & & & & & & & & & & & 19 & & \\
\hline & & & & & & & & & & & & & & & & .10 & & \\
\hline & $=0$ & 1 & 63 & 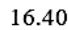 & 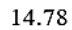 & 8 & $\delta$ & 3 & 10 & 9.29 & 0.4 & 8.00 & 7. & 0. & 6.50 & 6.12 & 5. & J. \\
\hline
\end{tabular}

Table 2. The denoising capacity interconnection of PSNR and the stable constant $T_{0}$ for pepper image

\begin{tabular}{|c|c|c|c|c|c|c|c|c|c|c|c|c|c|c|c|c|c|c|}
\hline \multirow{2}{*}{$\begin{array}{l}\text { Fix } \\
\text { Con }\end{array}$} & \multicolumn{18}{|c|}{ PSNR (dB) } \\
\hline & 5 & 10 & 15 & 20 & 25 & 30 & 35 & 40 & 45 & 50 & 55 & 60 & 65 & 70 & 75 & 80 & 85 & 90 \\
\hline 0.025 & 25.63 & 24.29 & 23.60 & 21.79 & 20.88 & 20.19 & 9.01 & 17.59 & 16.31 & 14.75 & 12.92 & 10.69 & 8.55 & 6.59 & 6.36 & 5.82 & 5.91 & 5.58 \\
\hline 0.050 & .43 & 2 & 6 & & & & & 2 & 16 & .33 & & & 29 & 02 & 26 & 84 & 77 & 5.63 \\
\hline 0.075 & .75 & 2 & .0 & 5 & 1 & 6 & 5 & 0 & 17.10 & & 8 & & 46 & 5 & 99 & 69 & 16 & 0.78 \\
\hline 0.100 & 4 & 3 & 3.58 & 1 & 23 & 6 & & 18 & 16.84 & & & 5 & .35 & 5 & 4 & 45 & 3 & 5.92 \\
\hline 0.125 & 8 & 3 & 8 & 1 & 6 & 6 & 2 & 1 & 16 & & & & 32 & 80 & 53 & 7.04 & 51 & 09 \\
\hline 0.150 & .60 & 3 & 2 & 7 & 1 & 8 & 0 & 1 & 15 & & & & 10.02 & 90 & 84 & 97 & 47 & 6.21 \\
\hline 175 & .56 & 3 & 4 & 3 & 20 & 19. & 3 & 5 & 1 & & & & 6 & 4 & 7.79 & 6.74 & 3 & 0. \\
\hline 200 & .36 & 2 & 2 & 0 & 20. & 18.5 & 3 & 1 & 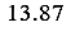 & & 9 & 10 & 6 & 8.04 & 7.17 & 5 & 18 & 5.7 \\
\hline 0.225 & .49 & 28.50 & 25.04 & .23 & 19.47 & 17.80 & 8 & 14.54 & 13. & 9 & 1 & 9.92 & 5 & 8.07 & 7.21 & 6.52 & 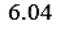 & 5. \\
\hline 0.250 & 2.13 & 27.37 & 4.09 & 21.36 & 18.87 & 17.2 & 1 & 14.19 & 12. & 2 & 5 & 9.58 & .14 & .94 & 7.30 & 66 & 9 & 5.73 \\
\hline 0.275 & 0.93 & 26.20 & 3.13 & .36 & 18.16 & 16.67 & 15.12 & 13.66 & 12.57 & 6 & 0 & & 69 & 6 & 7.47 & 6.70 & 8 & 5.82 \\
\hline 0.300 & 9.67 & 25.16 & 22.36 & .72 & 17.63 & 16.14 & 14.60 & 13.24 & 12.25 & 11.07 & 10. & 9. & 50 & 7.80 & 7.37 & 6.63 & 6.19 & 5.88 \\
\hline 0.325 & 8.69 & 24.26 & 1.52 & 9 & 17.02 & 15. & 14 & 13 & 11 & 10 & 4 & & 7 & 9 & 7.38 & 6.66 & 4 & 5.94 \\
\hline 0.350 & 27.75 & & 8 & & & & & & & & & & & 2 & 35 & 72 & & \\
\hline 75 & & & & & & & & & & & & & & 7. & 14 & 2 & & \\
\hline 100 & .05 & 2 & 3.8 & 1 & 15 & 13 & 2 & 11. & 10. & & 9 & & 7.94 & 7.4 & 6.98 & 6.62 & 6.2 & 5. \\
\hline 0.425 & .07 & 20.91 & 8.23 & 0 & 14 & 13. & 8 & 9 & 10. & 9.5 & 94 & & 7.81 & 7.32 & 6.90 & 6.56 & 6.2 & 5. \\
\hline 0.450 & 4.24 & 20.29 & .72 & 2 & 14. & 13. & 11.97 & 11.01 & 10. & 0 & 8.84 & 8.25 & 5 & 7.27 & 6.85 & 6.51 & 6.16 & 5. \\
\hline 0.475 & 23.60 & 19.77 & 17.28 & 15.48 & 13.98 & 12.81 & 11.78 & 10.88 & 10.07 & 9.36 & 8.77 & 8.20 & 7.72 & 7.25 & 6.84 & 6.50 & 6.15 & 5.85 \\
\hline 0.500 & 23.09 & 19.28 & 16.90 & 15.17 & 13.73 & 12.59 & 11.60 & 10.73 & 9.96 & 9.29 & 8.72 & 8.17 & 7.71 & 7.25 & 6.86 & 6.53 & 6.20 & 5.89 \\
\hline
\end{tabular}


- From these computer examining of Lena in Table 3, the optimized pre-specified constant $T_{0}$ is about $0.1125 \pm 0.0042$ or be fluctuated from 0.050 to 0.175 for making the finest DBAMF denoising capacity.

- From these computer examining of Airplane in Table 4, the optimized pre-specified constant $T_{0}$ is about $0.1097 \pm 0.0014$ or be fluctuated from 0.050 to 0.150 for making the finest DBAMF denoising capacity.

Table 3. The denoising capacity interconnection of PSNR and the stable constant $T_{0}$ for girl image

\begin{tabular}{|c|c|c|c|c|c|c|c|c|c|c|c|c|c|c|c|c|c|c|}
\hline \multirow{2}{*}{$\begin{array}{l}\text { Fix } \\
\text { Con }\end{array}$} & \multicolumn{18}{|c|}{ PSNR (dB) } \\
\hline & 5 & 10 & 15 & 20 & 25 & 30 & 35 & 40 & 45 & 50 & 55 & 60 & 65 & 70 & 75 & 80 & 85 & 90 \\
\hline 0.025 & 7.01 & 5.00 & .23 & .60 & .68 & 21.44 & 19.68 & 18.01 & 16.49 & 14.86 & 3.41 & 11.80 & 10.35 & 74 & 7.41 & 7.04 & .59 & 4.82 \\
\hline 050 & .19 & .68 & .24 & 26. & $2=$ & 23.03 & 0 & 17 & & 3 & & & & 20 & & 7.27 & & 505 \\
\hline 0.075 & 8 & 0 & .65 & 27. & & 23.26 & 20.60 & 37 & 16 & 1 & 13.61 & 1 & 5 & 4 & & 7.02 & 0 & 24 \\
\hline 0.100 & 5 & 34.48 & 6 & 5 & 26. & 8 & 20.28 & 18.40 & 16.93 & 6 & 13.68 & 9 & 4 & 1 & 8.64 & 7.30 & 6.52 & 5.29 \\
\hline 125 & 2 & 35.94 & 32.09 & 29.40 & 25 & 23. & 19.87 & 18.49 & 16.72 & 6 & 13.69 & 6 & 5 & 56 & 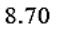 & .40 & 9 & 04 \\
\hline 0.150 & .20 & 4.97 & 3. & 28 & 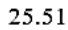 & 22 & 20.47 & 18.15 & 16.65 & 0 & & 6 & 0 & 53 & 75 & 7.50 & 9 & 5.15 \\
\hline 175 & 9 & 8 & 3 & 28 & 2. & 21. & 19. & 17.80 & 16 & 3 & & & & 42 & 1 & .43 & 1 & 5.27 \\
\hline 200 & 4 & 8 & 3 & 26 & 24 & 20. & 19. & 17.09 & 15 & 9 & & & 2 & 3 & 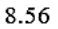 & 7.24 & 6.16 & 5.07 \\
\hline 0.225 & 2 & 32.00 & $2 \varepsilon$ & 25 & 23 & 20. & 18. & 16.27 & 15 & 13 & 12 & 11 & 10 & 8. & 8.42 & 6.93 & 6.04 & 4.96 \\
\hline 0.250 & 35.60 & 30.9 & 26.73 & 23. & 19. & 17. & 15. & 13.72 & 12 & 11. & 9 & 8 & 8 & 7.18 & 6. & 5.65 & 5. & 4.52 \\
\hline 0.275 & 34.43 & 30.14 & 26.37 & 22.88 & 19. & 17.87 & 15.51 & 13.79 & 12.50 & 10.98 & 9. & 9 & 7.96 & 7.31 & 6.6 & 5.66 & 5.04 & 4.86 \\
\hline 0.300 & 33.21 & 29.34 & 25.96 & 22.63 & 19.58 & 17.79 & 15.48 & 13.75 & 12.49 & 10.98 & 9. & 9.13 & 7.98 & 7.31 & 6.66 & 5.75 & 5.08 & 4.89 \\
\hline 0.325 & 33.01 & 29.18 & 25.84 & 22.55 & 19.53 & 17.76 & 15.45 & 13.74 & 12.47 & 10.98 & 9. & 9.13 & 00 & 7.32 & 6.48 & 5.98 & 5.11 & 4.56 \\
\hline 0.350 & 32.89 & 29.04 & 25.49 & 22.21 & 19.27 & 17.45 & 15.19 & 13.53 & 12.33 & 10.87 & 9. & 06 & 96 & 7.28 & 6.46 & 5.97 & 5.11 & 4.57 \\
\hline 0.375 & .82 & 28.32 & 24.74 & 21.61 & 18.59 & 16.85 & 14.73 & 13.15 & 12.02 & 10.58 & 9.6 & 77 & 7.81 & 7.17 & 6.38 & 5.67 & 5.07 & 4.55 \\
\hline 0.400 & .60 & 28.08 & 24.41 & 21.31 & 18.39 & 16.66 & 14.57 & 13.04 & 11.93 & 10.50 & 9. & 2 & 7.78 & 7.14 & 37 & 5.67 & 5.07 & 4.55 \\
\hline 0.425 & .38 & 27.80 & 24.14 & 21.00 & 18.15 & 16.38 & 14.39 & 12.89 & 11.79 & 10.38 & 9.50 & 64 & 2 & 07 & 32 & 5.64 & 05 & 4.54 \\
\hline 0.450 & 03 & 27.43 & 23.57 & 20.53 & 17.84 & 16.04 & 14.09 & 12.65 & 11.58 & 10.21 & 6 & 8.52 & 7.61 & 6.99 & 6.25 & 5.57 & 5.01 & 4.50 \\
\hline 0.475 & 31.24 & 27.10 & 22.89 & 19.73 & 17.18 & 15.44 & 13.55 & 12.20 & 11.20 & 9.87 & 9.0 & 8.26 & 7.40 & 6.82 & 6.14 & 5.47 & 4.94 & 4.46 \\
\hline 0.500 & 29.78 & 25.44 & 20.58 & 17.38 & 14.83 & 13.30 & 11.52 & 10.43 & 9.41 & 8.41 & 7.71 & 7.06 & 6.38 & 5.78 & 5.40 & 4.91 & 4.53 & 4.19 \\
\hline
\end{tabular}

Table 4. The denoising capacity interconnection of PSNR and the stable constant $T_{0}$ for LENA image

\begin{tabular}{|c|c|c|c|c|c|c|c|c|c|c|c|c|c|c|c|c|c|c|}
\hline \multirow{2}{*}{$\begin{array}{l}\text { Fix } \\
\text { cons. }\end{array}$} & \multicolumn{18}{|c|}{ PNSR (dB) } \\
\hline & 5 & 10 & 15 & 20 & 25 & 30 & 35 & 40 & 45 & 50 & 55 & 60 & 65 & 70 & 75 & 80 & 85 & 90 \\
\hline 0.025 & 5.34 & 25.82 & 24.23 & 23.44 & 22.50 & 24.58 & 20.21 & 19.06 & 17.53 & 15.85 & 13.87 & 10.72 & 8.43 & 7.53 & 6.05 & 6.09 & 6.18 & 5.69 \\
\hline 0.050 & 0.43 & 8.84 & 27.68 & 25.97 & 24.00 & 22.84 & 21.03 & 19.51 & 17.82 & & 14.15 & 11.24 & 9.22 & 8.16 & 6.19 & 6.13 & 6.20 & 5.69 \\
\hline .075 & 29.33 & 29.55 & 28.76 & 26.9 & & & & & & & & & & 5 & 6.87 & 6.43 & 31 & \\
\hline 0.10 & 36 & 25 & 28. & 27 & 6 & 22 & 2 & 7 & 5 & 7 & 2 & 1 & 10 & 0 & 8 & 9 & 8 & 5.15 \\
\hline 125 & 7 & 27.77 & 0 & 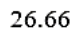 & 7 & 2 & 5 & 2 & 4 & 3 & 5 & 1 & 1 & 5 & 7.54 & 37 & 6 & 6.17 \\
\hline 0.150 & 2 & .48 & 3 & 2 & 9 & 20. & 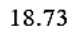 & 4 & 6 & 4 & 0 & & 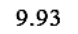 & 7 & 7 & 76 & 0 & 6.09 \\
\hline 0.17 & .03 & 8 & 27 & 2 & 2 & 19 & 1 & 39 & 5 & & & & 36 & 12 & 7.55 & 6.77 & 23 & 5.95 \\
\hline 0.200 & $3 \varepsilon$ & & & & & & & & & & & & 4 & 00 & 29 & 6.61 & 6 & 5.83 \\
\hline 0.22 & 36. & 29 & 25 & 2 & & & & & & & & & 1 & 98 & 8 & 6.71 & 22 & 5.82 \\
\hline 0.25 & 34 & $2 \varepsilon$ & & & & & & & & & & & & 99 & 5 & 7.01 & 20 & 5.97 \\
\hline 0.27 & 32. & 26. & 22 & & & & & & & & & & 3 & 08 & 7 & 6.95 & 6 & 6.08 \\
\hline 0.30 & 30. & & & & & & & & & & & & & 12 & 5 & 7.01 & 5 & 5.79 \\
\hline 0.32 & 28.82 & 24.31 & 20 & & 0 & & & 1 & 1 & & & & 0 & 07 & 7.22 & 7.05 & 27 & 6.07 \\
\hline 0.35 & 27.60 & 23.23 & 19.92 & & 16.05 & 14. & & 12.18 & 11 & & & & & 7.92 & 7.16 & 6.76 & 3 & 6.08 \\
\hline 0.37 & 26.60 & 22.17 & 19.09 & 17. & 15.34 & 14.05 & 12. & 11.71 & 10 & & & & 7 & 7.59 & 7.09 & 6.74 & 6.40 & 6.09 \\
\hline 0.400 & 25.65 & 21.37 & 18.49 & 16.55 & 14.92 & 13.69 & 12.45 & 11.47 & 10. & 9 & 9.0 & & 0 & 7.53 & 7.06 & 6.71 & 6.39 & 6.09 \\
\hline 0.425 & 24.93 & 20.72 & 17.90 & & 14.57 & 13.39 & 12.22 & 11.29 & 10. & 9. & .0 & & 6 & 7.49 & 7.04 & 6.69 & 6.36 & 6.05 \\
\hline 0.450 & 24.28 & 20.16 & 17.43 & 15. & 14.23 & 13.11 & 12.00 & 11.09 & 10 & 9.57 & 8.93 & & 7.91 & 7.45 & 7.02 & 6.67 & 6.33 & 6.02 \\
\hline 0.475 & 23.43 & 19.49 & 16.88 & 15. & 13.81 & 12.76 & 11. & 10.86 & 10.06 & & & & 5 & 7.42 & 7.01 & 6.67 & 6.34 & 6.04 \\
\hline 0.500 & 22.79 & 18.88 & 16.39 & 14.83 & 13.45 & 12.45 & 11.49 & 10.67 & 9.89 & 9.31 & 8.73 & 2.18 & 7.82 & 7.41 & 7.02 & 6.70 & 6.39 & 6.08 \\
\hline
\end{tabular}

\subsection{The experimental investigation of image denoising method found on DBAMF}

This analyzed report focuses to examine the computational scrutiny of image denoising method found on DBAMF under SPN surrounding. In this examining of the DBAMF denoising capacity, four analyzed images, which are contained of Airplane, Pepper, Girl and Lena, are used to analyzed by initially adding synthesized SP noise for creating numerous distorted portraits. Later, all distorted portraits are repaired for obtaining the finest quality and best PSNR by executing the image denoising method found on DBAMF. From the above examining, the denoising methods by applying AMF [7, 25] and DBAMF produce the finest quality and best PSNR than other denoising methods for instant SMF and Gaussian filter. However, the DBAMF has marginally improved than AMF from that fact that the DBAMF is initially developed solely for random magnitude impulsive noise (RMIN) but the AMF is developed solely for SPN. From these computer examining of the denoising capacity in Table 5(a) for Lena and Pepper and Table 5(b) for Girl and Airplane, althrogh the DBAMF denoising method is originally desired for RVIN, the DBAMF denoising method can provide the fine results (the denoised images with high quality). From these inverstigation, the DBAMF method and adaptive median filter (AMF) can produce the denoised image with finer quality and high PSNR, which is confronted with the other denoised images, which is computed by standard median filter (SMF) and Gaussian Filter. Due to circumspection of sheet of paper, some 
graphical results (of Lena image at 10\% and 20\%) of the denoised DBAMF method and other denoised methods are shown in Figure 4.

Table 5 (a). The analysis report of the denoising capacity of DBAMF under SPN surrounding

\begin{tabular}{|c|c|c|c|c|c|c|}
\hline \multirow{3}{*}{$\frac{\text { SPN }}{\text { Operated images }}$} & \multicolumn{6}{|c|}{ PNSR (dB) } \\
\hline & \multirow{2}{*}{ Noise density } & \multirow{2}{*}{ LR image } & \multicolumn{4}{|c|}{ Noise suppressing technique } \\
\hline & & & Median (3x3) & Mean $(3 \times 3)$ & AMF & DBA MF \\
\hline \multirow{18}{*}{$\begin{array}{c}\text { Lena } \\
(256 \times 256)\end{array}$} & $\mathrm{D}=0.05$ & 18.7139 & 31.6421 & 22.4181 & 36.0907 & 40.0318 \\
\hline & $\mathrm{D}=0.10$ & 15.6564 & 30.7076 & 19.3812 & 35.3032 & 34.4817 \\
\hline & $\mathrm{D}=0.15$ & 13.8274 & 29.2982 & 17.5385 & 33.7454 & 29.3024 \\
\hline & $\mathrm{D}=0.20$ & 12.6389 & 27.6257 & 16.3208 & 32.1558 & 27.1381 \\
\hline & $\mathrm{D}=0.25$ & 11.6783 & 25.4101 & 15.3526 & 29.8105 & 24.7729 \\
\hline & $\mathrm{D}=0.30$ & 10.8971 & 23.6811 & 14.5829 & 27.9141 & 22.9755 \\
\hline & $\mathrm{D}=0.35$ & 10.2240 & 20.8127 & 13.8785 & 25.6654 & 21.0287 \\
\hline & $\mathrm{D}=0.40$ & 9.6481 & 19.0080 & 13.2479 & 23.7903 & 19.5071 \\
\hline & $\mathrm{D}=0.45$ & 9.0745 & 16.8389 & 12.6598 & 21.5949 & 17.8200 \\
\hline & $\mathrm{D}=0.50$ & 8.6553 & 15.4758 & 12.2146 & 20.5725 & 16.1734 \\
\hline & $\mathrm{D}=0.55$ & 8.2118 & 13.8573 & 11.7609 & 19.4896 & 14.1775 \\
\hline & $D=0.60$ & 7.7813 & 12.3280 & 11.2939 & 18.1747 & 11.7120 \\
\hline & $\mathrm{D}=0.65$ & 7.4884 & 11.3251 & 11.0012 & 17.7283 & 10.3838 \\
\hline & $\mathrm{D}=0.70$ & 7.1697 & 10.2861 & 10.6509 & 17.1153 & 8.9514 \\
\hline & $\mathrm{D}=0.75$ & 6.8497 & 9.1271 & 10.2599 & 16.5388 & 7.5456 \\
\hline & $\mathrm{D}=0.80$ & 6.5846 & 8.3331 & 10.0057 & 16.4554 & 7.0520 \\
\hline & $\mathrm{D}=0.85$ & 6.3241 & 7.5344 & 9.7338 & 16.4230 & 6.4819 \\
\hline & $\mathrm{D}=0.90$ & 6.0604 & 6.8241 & 9.4356 & 16.5352 & 6.1742 \\
\hline Pepper & $\mathrm{D}=0.05$ & 18.4752 & 32.2578 & 22.1408 & 37.1145 & 37.5975 \\
\hline \multirow{17}{*}{$(256 \times 256)$} & $\mathrm{D}=0.10$ & 15.3798 & 30.6116 & 19.0677 & 36.0391 & 32.8628 \\
\hline & $\mathrm{D}=0.15$ & 13.5570 & 28.8470 & 17.2234 & 33.6095 & 28.5840 \\
\hline & $\mathrm{D}=0.20$ & 12.3593 & 26.5888 & 15.9804 & 31.6485 & 25.9117 \\
\hline & $\mathrm{D}=0.25$ & 11.3929 & 24.2073 & 14.9986 & 29.4205 & 23.6700 \\
\hline & $\mathrm{D}=0.30$ & 10.6242 & 22.0663 & 14.1748 & 26.7650 & 21.7606 \\
\hline & $\mathrm{D}=0.35$ & 9.9742 & 20.3774 & 13.5209 & 25.5249 & 20.3507 \\
\hline & $\mathrm{D}=0.40$ & 9.3998 & 18.4321 & 12.9076 & 23.4995 & 18.4004 \\
\hline & $\mathrm{D}=0.45$ & 8.8599 & 16.6168 & 12.3275 & 21.7177 & 17.0967 \\
\hline & $\mathrm{D}=0.50$ & 8.3843 & 14.8506 & 11.8117 & 20.2203 & 15.3313 \\
\hline & $\mathrm{D}=0.55$ & 7.9930 & 13.4655 & 11.3720 & 19.0894 & 13.5815 \\
\hline & $\mathrm{D}=0.60$ & 7.6189 & 12.0128 & 10.9563 & 18.1116 & 11.9462 \\
\hline & $\mathrm{D}=0.65$ & 7.2684 & 10.8920 & 10.5158 & 17.3657 & 10.3517 \\
\hline & $\mathrm{D}=0.70$ & 6.9246 & 9.7704 & 10.2039 & 16.5923 & 8.9034 \\
\hline & $\mathrm{D}=0.75$ & 6.6418 & 8.8751 & 9.8955 & 16.2338 & 7.8407 \\
\hline & $\mathrm{D}=0.80$ & 6.3710 & 8.0166 & 9.252 . & 16.0896 & 7.0426 \\
\hline & $\mathrm{D}=0.85$ & 6.1097 & 7.2402 & 9.2949 & 16.0498 & 6.5095 \\
\hline & $\mathrm{D}=0.90$ & 5.8582 & 6.5767 & 9.0214 & 16.2932 & 6.2081 \\
\hline
\end{tabular}

Table 5 (b). The analysis report of the denoising capacity of DBAMF under SPN surrounding

\begin{tabular}{|c|c|c|c|c|c|c|}
\hline \multirow{3}{*}{$\frac{\text { SPN }}{\text { Operated images }}$} & \multicolumn{6}{|c|}{ PNSR $(\mathrm{dB})$} \\
\hline & \multirow[t]{2}{*}{ Noise density } & \multirow[t]{2}{*}{ LR image } & \multicolumn{4}{|c|}{ Noise suppressing technique } \\
\hline & & & Median (3x3) & Mean $(3 \times 3)$ & AMF & DBA MF \\
\hline \multirow{18}{*}{$\begin{array}{c}\text { Girl } \\
(256 \times 256)\end{array}$} & $\mathrm{D}=0.05$ & 16.4490 & 32.4867 & 20.0454 & 37.5895 & 39.2037 \\
\hline & $\mathrm{D}=0.10$ & 13.6890 & 31.5583 & 17.2530 & 36.9197 & 35.9413 \\
\hline & $\mathrm{D}=0.15$ & 11.9287 & 27.6179 & 15.3515 & 34.818 & 32.0931 \\
\hline & $\mathrm{D}=0.20$ & 10.6567 & 25.5153 & 13.9593 & 32.0437 & 29.3961 \\
\hline & $\mathrm{D}=0.25$ & 9.5498 & 22.9614 & 12.7148 & 29.6074 & 26.069 \\
\hline & $\mathrm{D}=0.30$ & 8.8677 & 20.7738 & 11.9599 & 27.6930 & 23.2608 \\
\hline & $\mathrm{D}=0.35$ & 8.0984 & 18.4410 & 11.0501 & 34.9709 & 20.5962 \\
\hline & $\mathrm{D}=0.40$ & 7.5798 & 1635146 & 10.4543 & 23.3736 & 18.4867 \\
\hline & $\mathrm{D}=0.45$ & 7.0728 & 14.8145 & 9.8471 & 21.8119 & 16.9252 \\
\hline & $\mathrm{D}=0.50$ & 6.5712 & 13.0319 & 9.2367 & 20.1712 & 15.1631 \\
\hline & $\mathrm{D}=0.55$ & 6.2085 & 11.8226 & 8.7895 & 19.2184 & 13.6912 \\
\hline & $\mathrm{D}=0.60$ & 5.8609 & 10.4981 & 8.3590 & 18.4518 & 12.2929 \\
\hline & $\mathrm{D}=0.65$ & 5.4832 & 3.1396 & 7.8712 & 17.2740 & 10.9353 \\
\hline & $\mathrm{D}=0.70$ & 5.1311 & 8.0463 & 7.4271 & 16.7334 & 9.5595 \\
\hline & $\mathrm{D}=0.75$ & 4.8712 & 7.1994 & 7.0814 & 16.2921 & 8.703 \\
\hline & $\mathrm{D}=0.80$ & 4.5674 & 6.2520 & 6.6881 & 16.2795 & 7.5002 \\
\hline & $\mathrm{D}=0.85$ & 4.3054 & 5.4218 & 6.3340 & 16.5924 & 6.5222 \\
\hline & $\mathrm{D}=0.90$ & 4.0573 & 4.7465 & 5.9986 & 16.7463 & 5.2931 \\
\hline \multirow{18}{*}{$\begin{array}{l}\text { Airplane } \\
(256 \times 256)\end{array}$} & $\mathrm{D}=0.05$ & 17.9498 & 31.4106 & 21.5802 & 36.6063 & 36.5023 \\
\hline & $\mathrm{D}=0.10$ & 14.8320 & 29.6532 & 18.4426 & 34.6311 & 31.5421 \\
\hline & $D=0.15$ & 13.1197 & 28.3176 & 16.6870 & 33.5561 & 29.9163 \\
\hline & $\mathrm{D}=0.20$ & 11.8045 & 26.4356 & 15.3181 & 31.3844 & 26.2166 \\
\hline & $\mathrm{D}=0.25$ & 10.9272 & 24.4147 & 14.3866 & 29.5029 & 25.0114 \\
\hline & $\mathrm{D}=0.30$ & 10.0510 & 21.8862 & 13.4526 & 27.1347 & 22.1793 \\
\hline & $\mathrm{D}=0.35$ & 9.4325 & 19.6835 & 12.7646 & 26.0118 & 20.5242 \\
\hline & $\mathrm{D}=0.40$ & 8.8735 & 17.6412 & 12.1397 & 23.0147 & 18.5963 \\
\hline & $\mathrm{D}=0.45$ & 8.3344 & 15.8686 & 11.5224 & 21.2768 & 17.0442 \\
\hline & $\mathrm{D}=0.50$ & 7.8600 & 14.2697 & 11.0091 & 19.6201 & 15.4295 \\
\hline & $\mathrm{D}=0.55$ & 7.4696 & 12.8823 & 10.5769 & 18.6408 & 13.9249 \\
\hline & $\mathrm{D}=0.60$ & 7.0920 & 11.5290 & 10.1202 & 17.6586 & 12.3281 \\
\hline & $D=0.65$ & 6.7276 & 10.4080 & 9.7008 & 16.9400 & 10.9416 \\
\hline & $\mathrm{D}=0.70$ & 6.4028 & 9.3041 & 9.3238 & 16.2514 & 9.8385 \\
\hline & $\mathrm{D}=0.75$ & 6.1274 & 8.3797 & 9.0020 & 15.9223 & 8.6814 \\
\hline & $\mathrm{D}=0.80$ & 5.8647 & 7.5835 & 8.6893 & 15.7428 & 7.9942 \\
\hline & $\mathrm{D}=0.85$ & 5.5768 & 6.7043 & 8.3346 & 15.8098 & 6.9951 \\
\hline & $\mathrm{D}=0.90$ & 5.3335 & 6.0278 & 8.0381 & 16.0834 & 6.3296 \\
\hline
\end{tabular}




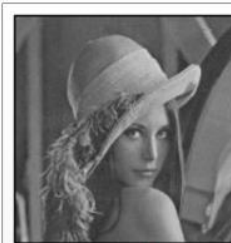

(a-1)

Original Image

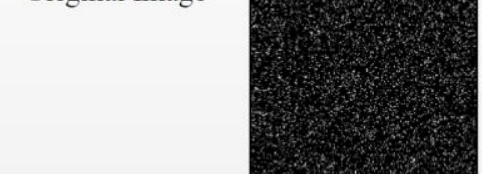

$(\mathrm{a}-2)(\mathrm{D}=10 \%)$

Corrupted Image

$3 \times 3$ Mean Filter
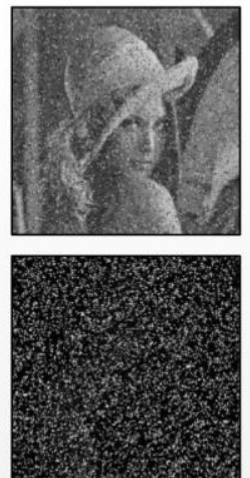

(a-4)
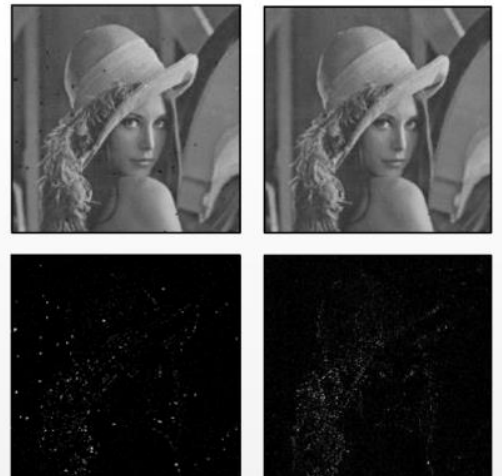

(a-5)

DBAMF

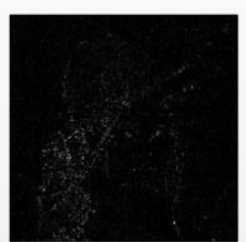

(a-6)

(a)

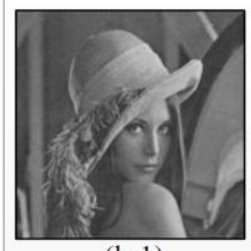

(b-1)

Original Image

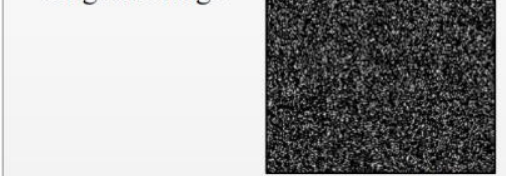

(b-2) $(\mathrm{D}=20 \%)$

Corrupted Image
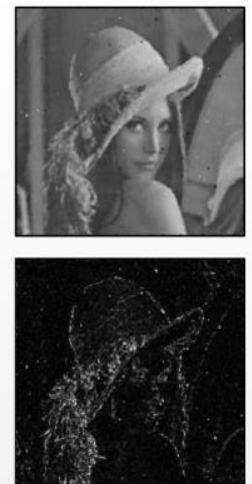

(b-3)

$3 \times 3$ Median Filter
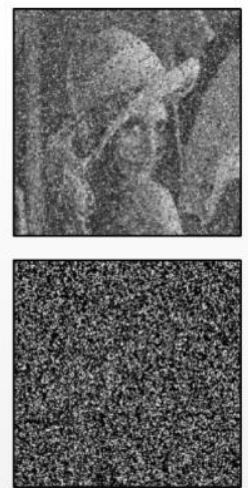

(b-4)

$3 \times 3$ Mean Filter
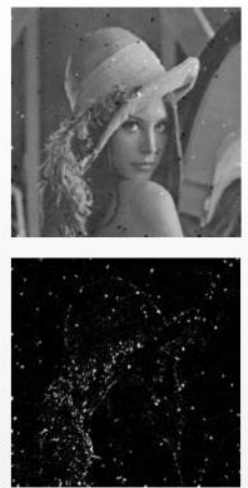

(b-5)

DBAMF
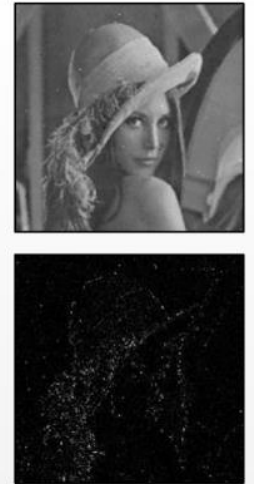

(b-6)

AMF Filter $(\mathrm{PSNR}=16.3208 \mathrm{~dB})(\mathrm{PSNR}=27.1381 \mathrm{~dB})(\mathrm{PSNR}=32.1558 \mathrm{~dB})$

(b)

Figure 4. The analysis report of noise repairing method of the DBAMF

\section{CONCLUSION}

This analyzed report focuses to examine the similarity capacity of denoising method found on DBAMF for diverse SPN Surrounding. In order to examine the denoising capacity and its obstruction of the denoising method found on DBAMF, the four original digital images, comprised of Airplane, Pepper, Girl and Lena, are examined in these computational simulation for SPN surrounding by initially contaminating the SPN with diverse intensity. The first constribution of this report is the optimized stable constant, which is determined from computer simulation at SPN surrounding. Later, the second constribution of this report is the overall capacity of denoising method found on DBAMF, confronted with the other denoised images (SMF and gaussian filter and adaptive median filter (AMF)) under the SPN with diverse intensity.

\section{ACKNOWLEDGEMENTS}

The research project was funded by Assumption University. 


\section{REFERENCES}

[1] A. Awad, "Removal of fixed-valued impulse noise based on probability of existence of the image pixel," International Journal of Electrical \& Computer Engineering, vol. 8, no. 4, pp. 2106-2114, 2018.

[2] H. Hwang and R. A. Haddad, "Adaptive median filters new algorithms and results," IEEE Transactions of Image Processing, vol. 4, pp. 4, pp. 499-502, 1994.

[3] Kornkamol Thakulsukanant and Vorapoj Patanavijit, "Simulation evaluation of noise suppressing technique based on decision based adaptive median filter for salt \& pepper noise," 16th International Conference on Electrical Engineering/Electronics, Computer, Telecommunications and Information Technology, pp. 585-588, 2019.

[4] K. Arun Sai, K. Ravi, "An efficient filtering technique for denoising colour images," International Journal of Electrical and Computer Engineering (IJECE), vol. 8, no. 5, pp. 3604-3608, 2018.

[5] R. H. Chan, C-W Ho and M. Nikilova, "Salt \& pepper noise removal by median-type noise detectors and detailpreserving regularization," IEEE Transactions of Image Processing, vol. 14, no. 10, pp. 1479-1485, 2005.

[6] V. Patanavijit, "The bilateral denoising performance influence of window," Spatial and Radiometric Variance, 2015.

[7] V. Patanavijit, "Performance analysis of denoising algorithm based on adaptive median filter under unsystematic intensity impulse and salt \& pepper noise," International Electrical Engineering Congress, pp. 1-4, 2018.

[8] W. K. Pratt, "Median filtering," Tech. Rep., Image Proc. Inst., Univ. Southern California, Los Angeles, 1975.

[9] Yiqiu Dong, Raymond H. Chan, and Shufang Xu, "A detection statistic for random-valued impulse noise," IEEE Transactions on Image Processing, vol. 16, no. 4. pp. 1112-1120, 2007

[10] Om Prakash Verma and Nitin Sharma, "Intensity preserving cast removal in color images using particle swarm optimization," International Journal of Electrical \& Computer Engineering, vol. 7, no. 5, pp. 2581-2595, 2017.

[11] Madina Hamiane and Fatema Saeed, "SVM classification of MRI brain images for computer-assisted diagnosis, International Journal of Electrical and Computer Engineering, vol. 7, no. 5, pp. 2555-2564, 2017.

[12] A. S. M. Shafi, et.al., "Decomposition of color wavelet with higher order statistical texture and convolutional neural network features set based classification of colorectal polyps from video endoscopy," International Journal of Electrical and Computer Engineering, vol. 10, no. 3, pp. 2986-2996, 2020.

[13] S. Bagchi, et.al, "Image processing and machine learning techniques used in computer-aided detection system for mammogram screening-A review," International Journal of Electrical and Computer Engineering, vol. 10, no. 3, pp. 2336-2348, 2020.

[14] César G. Pachón-Suescún, Carlos J. Enciso-Aragón, Robinson Jiménez-Moreno, "Robotic navigation algorithm with machine vision," International Journal of Electrical and Computer Engineering, vol. 10, no. 2, pp. 1308-1316, 2020.

[15] D. Kesrarat, et.al, "A novel elementary spatial expanding scheme form on sisr method with modifying geman \& mcclure function," TELKOMNIKA Telecommunication, Computing, Electronics and Control, vol. 17, no.5, 2019.

[16] V. Patanavijit, "Denoising performance analysis of adaptive decision based inverse distance weighted interpolation (DBIDWI) algorithm for salt and pepper noise," Indonesian Journal of Electrical Engineering and Computer Science, vol. 15, no. 2, pp. 804-813, 2019.

[17] V. Kishorebabu1, et.al., "An adaptive decision based interpolation scheme for the removal of high density salt and pepper noise in images," EURASIP Journal on Image and Video Processing, vol. 2017, no. 1, 2017.

[18] V. Patanavijit, et.al, "The statistical analysis of random-valued impulse noise detection techniques based on the local image characteristic: ROAD, ROLD and RORD," Indonesian Journal of Electrical Engineering and Computer Science, vol. 15, no. 1, pp.749-803, 2019.

[19] Y. Dong, et.al, “A detection statistic for random-valued impulse noise," IEEE Transactions on Image Processing, vol. 16, no. 4, pp. 1112-1120, 2007.

[20] H. Yu, et.al, "An efficient procedure for removing random-valued impulse noise in images," IEEE Signal Processing Letters, vol. 15, pp. 922-925, 2008.

[21] V. Jayaraj and D. Ebenezer, "A New Switching-BasedMedian Filtering Scheme and Algorithm for Removal of High-Density Salt and Pepper Noise in Images," EURASIP JASP, 2010.

[22] Ali S. Awad, "Standard Deviation for Obtaining the Optimal Direction in the Removal of Impulse Noise," IEEE Signal Letters, 2011.

[23] V. Patanavijit and K. Thakulsukanant, "Simulated Evaluation of New Switching Based Median Filter for Suppressing SPN and RVIN," Indonesian Journal of Electrical Engineering and Computer Science, Vol. 15, No. 2, Aug. 2019.

[24] R. C. Gonzalez and R. E. Woods, “Digital Image Processing," Prentice Hall, 2nd edition, NJ, USA, 2002.

[25] V. R. Vijaykumar and P. Jothibasu, "Decision Based Adatpvive Median Filter to Remove Blotches, Scratches, Streaks, Stripes and Impulse Noise in Images," 2010 IEEE International Conference on Image Processing, Hong Kong, pp. 117-120, September 2010. 